\title{
Interactive comment on "The relevance of mid-Holocene Arctic warming to the future" by Masakazu Yoshimori and Marina Suzuki
}

\author{
Masakazu Yoshimori and Marina Suzuki \\ masakazu@aori.u-tokyo.ac.jp
}

Received and published: 15 January 2019

Thank you very much for carefully reading the manuscript and for bringing up some important points. Perspective on the revision is provided below.

As to the discussion, we will revise the manuscript and place more emphasis on why we consider the knowledge of mid-Holocene Arctic warming is useful when future Arctic warming projections are made. What we consider the most important point is that the two different time periods ( $\mathrm{MH}$ and future) are relevant through similarities of dominant processes in shaping the Arctic warming even though the radiative forcing and climate response are not alike. It is an indirect relevance, but this view has not been explored much previously, particularly for the Arctic warming. It is not a priori trivial whether the 
dominant processes are common in response to two different climate forcers among many processes, and we show a link in mechanism between the two climates.

As to the conclusion, we will revise the manuscript so that it highlights more on what is new here. As the reviewer pointed out, the way it was rewritten may be too generalized with less emphasis on specific findings here. As the reviewer says, the phrase "improvement of the ability of the model to simulate the past will increase the confidence in their ability to simulate the future" may have been used repeatedly, but it is often used in an intuitive context, rather than with rigorous analysis, particularly for the Arctic warming. What we consider new findings here are 1) the similarities in processes responsible for the Arctic warming between $\mathrm{MH}$ and future despite of very different radiative forcing pattern; and 2) those processes are also contribute to the model spread in the Arctic warming between the two periods. Mechanism of $\mathrm{MH}$ Arctic warming and future in multi-models have not been investigated in parallel previously and to the extent conducted here. In addition, the similarities in processes are not shown previously. Furthermore, the warming processes are detailed by separating the sea surface temperature and sea ice surface temperature changes in Fig. 7 which adds clear physical understanding to the conventional surface energy balance feedback analysis. We will make the conclusion sharper.

We will add Data Availability section. We will also make the main part of the program used in the analysis for Fig. 5 publicly available.

Specific comments.

$>$ P1-129: is it solar forcing?

Marshall et al. (2014) suggests stratospheric ozone forcing. To be specific, we will change it to "stratospheric ozone change and cloud feedback play roles".

>P2-I1 : I assume that the 'scenario' refers to RCP scenarios. This should be made clear.

Interactive

comment

Printer-friendly version

Discussion paper 
Change will be made to "RCP scenario".

>P4-I16 and P7-I13 : there is a reference to Sect. 3a, which does not exist (at least as such).

"3a" should be "3.1". Thank you.

>P6-133 : According to my reading of the figure, the simulated warming only occurs in the northern North Atlantic and Arctic oceans, where there is no data. It is therefore very difficult to say if it is under- or over-estimated. Or do the authors call 'warming' the negative values in the figure?

We will change it to "the warming indicated by the reconstruction is not captured by the model mean in January as well as in the annual mean."

>P7-I16 : 'plays an important role'. According to my reading, this is only true in JJA.

We will change it to "plays an important role in June-July-August (and in SeptemberOctober in opposite sign)".

>P7-I32 : 'exhibits a large contribution'. This does not really seem to be the case for $\mathrm{MH}$.

The reviewer is correct. We will clarify this point.

>P8-19: could the authors make the label coherent (Dtas in the text, Dta in the figure).

The correction will be made to the text.

>P10-I11: PMIP3 instead of PMIM3

Will be corrected. Thank you.

>P10-I22: The authors should make their conclusion readable by itself. It should be said that the Arctic warming is for the future (under RCP4.5).

Printer-friendly version

We will revise the conclusion taking the reviewer's comment into account. 
>P10-133 : 'seeking possible analogues between physical processes in the past and future climate'. Do the authors mean that the climate processes are time dependent? I thought that they were based on basic physical principles valid through time. Moreover, as we do not know the future climate it is hard to look for analogues there and then.

While physical principles are same throughout the time, what we meant was that it is not trivial that the dominating processes for the climate variations are the same for different climate forcing and change cases. We will rephrase the sentence.

$>$ P15: A reference is missing here.

We will add the reference.

$>P 21$ : The figure is misleading because the $\mathrm{Y}$-axis (scale) is not the same for $\mathrm{MH}$ and RCP4.5.

We will add the note on the caption. The difference in magnitude does not preclude the use of these two different time periods, and rather it is of interest that such different climate responses still share the similar dominant processes.

>P23-14 : I do not see two (black and blue dashed) lines. Are they exactly superimposed? In that case, this should be mentioned in the caption.

They are black polygonal solid-line and blue polygonal dashed-line. We will make the caption more precise.

>P24-26: Figures 6-8 are not using the same number of models. (1) the name of the models used should be mentioned. (2) Not using all the models (and not always the same models) may introduce a bias in the interpretation. Would the conclusion be the same if only the models (and their outputs) available for all the figures were used?

(1) All model names were given in Table 2, but the models used for Fig. 7 was only written in text. We will refer Table 2 for Figs. 6 and 8, and write names explicitly for Fig. 7 in the caption. (2) The main results shown in Figs. 5 and 10 are benefitted

Printer-friendly version

Discussion paper 
the most by using the models as many as possible (10 models), but all variables are available only for 5 models: 5 models are missing for Fig. 7 and 1 model is missing for Fig. 8 (It was mistakenly written that 2 models were missing in the caption of Fig. 8. We will correct it). We checked the consistency of Figs. 5, 7 and 8 by reducing the model numbers to 5 . Figs. 5 and 8 were not qualitatively affected by this reduction. We also checked all figures by reducing the model numbers to 5: a few terms lost their statistical significance in Fig. 10, but the conclusion remains the same.

Interactive comment on Clim. Past Discuss., https://doi.org/10.5194/cp-2018-175, 2018. 